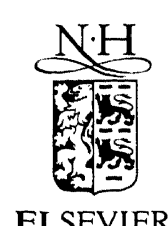

ELSEVIER

\title{
Condition improvement for point relaxation in multigrid, subsonic Euler-flow computations *
}

\author{
Barry Koren
}

CWI, P.O. Box 94079, 1090 GB Amsterdam, Netherlands

\begin{abstract}
Insight is given into the conditions of derivative matrices to be inverted in point-relaxation methods for 1-D and 2-D, first-order upwind-discretized Euler equations. Speed regimes are found where ill-conditioning of these matrices occurs; 1-D flow equations appear to be less well conditioned than 2-D flow equations. The ill-conditioning is easily improved by adding regularizing matrices to the derivative matrices. A smoothing analysis is made of point Gauss-Seidel relaxation applied to discrete Euler equations conditioned by such an additive matrix. The method is successfully applied to a very low-subsonic, steady, 2-D stagnation flow.
\end{abstract}

Keywords: Subsonic flows; Euler equations; Multigrid methods; Conditioning matrices; Convergence

\section{Introduction}

The present paper is a sequel to [1] and [3]. In [1] a basic multigrid solution method for steady, first-order upwind-discretized Euler equations was presented. In [3] some adaptations to this basic method were presented for high-supersonic (hypersonic) flow computations. In the present paper some necessary adaptations are presented for low-subsonic flow computations. By the latter adaptations, the range of Mach numbers over which the multigrid method can be applied successfully, is made as wide as necessary for practical purposes. Of course, in practice, first-order solution accuracy is insufficient. We know already that higher-order discrete equations can simply be solved by putting an additional iteration (a defect correction iteration) around the multigrid iteration (see e.g. [2]). The present paper is intended to complete the applicability of the multigrid method from [1,3].

In the zero-Mach-number limit, point-relaxation methods for solving first-order upwind-discretized Euler equations may suffer from ill-conditioning of the corresponding derivative

\footnotetext{
The research reported was performed in the framework of the BRITE-EURAM Aeronautics R\&D Programme of the European Communities (Contract No. AER2-CT92-0040).
} 
matrices to be inverted (i.e. the local, absolute-eigenvalue matrices, not the Jacobians). To illustrate this, we start by considering the perfect-gas, steady, 1-D Euler equations

$$
\frac{\mathrm{d} f(Q)}{\mathrm{d} x}=0
$$

with $Q$ the conservative state vector

$$
Q=\left(\begin{array}{c}
\rho \\
\rho u \\
\rho e
\end{array}\right), \quad e=\frac{1}{\gamma-1} \frac{p}{\rho}+\frac{1}{2} u^{2}
$$

and $f(Q)$ the corresponding flux vector

$$
f(Q)=\left(\begin{array}{c}
\rho u \\
\rho u^{2}+p \\
\rho u(e+p / \rho)
\end{array}\right) .
$$

Linearization of (1a) with respect to the conservative variables and, following [5], transformation from conservative variables $Q$ to non-conservative (entropy) variables $q$,

$$
\mathrm{d} q \equiv\left(\begin{array}{c}
(1 / \rho c) \mathrm{d} p \\
\mathrm{~d} u \\
\mathrm{~d} p-c^{2} \mathrm{~d} \rho
\end{array}\right)
$$

yields the analytically tractable form

$$
\begin{aligned}
& A \frac{\mathrm{d} q}{\mathrm{~d} x}=0 \\
& A=\frac{\mathrm{d} q}{\mathrm{~d} Q} \frac{\mathrm{d} f}{\mathrm{~d} Q} \frac{\mathrm{d} Q}{\mathrm{~d} q}=\left(\begin{array}{lll}
u & c & 0 \\
c & u & 0 \\
0 & 0 & u
\end{array}\right), \\
& \frac{\mathrm{d} f}{\mathrm{~d} Q}=\left(\begin{array}{ccc}
0 & 1 & 0 \\
\frac{\gamma-3}{2} u^{2} & (3-\gamma) u & \gamma-1 \\
\frac{\gamma-2}{2} u^{3}-\frac{1}{\gamma-1} u c^{2} & \frac{(3-2 \gamma)}{2} u^{2}+\frac{1}{\gamma-1} c^{2} & \gamma u \\
\frac{\rho}{c} & 0 & -\frac{1}{c^{2}} \\
\frac{\rho u}{c} & \rho & -\frac{u}{c^{2}} \\
\frac{1}{2} \frac{\rho u^{2}}{c}+\frac{1}{\gamma-1} \rho c & \rho u & -\frac{1}{2} \frac{u^{2}}{c^{2}}
\end{array}\right)
\end{aligned}
$$


For simplicity we assume $A$ to be constant and proceed by considering a first-order upwind, cell-centered finite-volume discretization of (3a). Then the discrete equation in cell $\Omega_{i}$ reads

$$
A^{+}\left(q_{i}-q_{i-1}\right)+A^{-}\left(q_{i+1}-q_{i}\right)=0,
$$

with $i$ running in the positive $x$-direction, and with $A^{+}$and $A^{-}$the matrices corresponding to the positive and negative eigenvalues of $A$ :

$$
A^{+}=R_{A} \Lambda_{A}^{+} R_{A}^{-1}, \quad A^{-}=R_{A} \Lambda_{A}^{-} R_{A}^{-1} .
$$

With $\Lambda_{A}=\operatorname{diag}(u-c, u, u+c)$ it follows that

$$
R_{A}=\left(\begin{array}{rrr}
1 & 0 & 1 \\
-1 & 0 & 1 \\
0 & 1 & 0
\end{array}\right)
$$

and hence for subsonic flow in the positive $x$-direction, $0<u<c$ :

$$
A^{+}=\frac{1}{2}\left(\begin{array}{ccc}
u+c & u+c & 0 \\
u+c & u+c & 0 \\
0 & 0 & 2 u
\end{array}\right), \quad A^{-}=\frac{1}{2}\left(\begin{array}{ccc}
u-c & c-u & 0 \\
c-u & u-c & 0 \\
0 & 0 & 0
\end{array}\right)
$$

Applying point Gauss-Seidel relaxation to find the solution $q_{i}$ of (4), for successively downstream and upstream relaxation sweeps, we get the iteration formulae

$$
\begin{aligned}
& |A|\left(q_{i}^{n+1}-q_{i}^{n}\right)=-A^{+}\left(q_{i}^{n}-q_{i-1}^{n+1}\right)-A^{-}\left(q_{i+1}^{n}-q_{i}^{n}\right), \\
& |A|\left(q_{i}^{n+2}-q_{i}^{n+1}\right)=-A^{+}\left(q_{i}^{n+1}-q_{i-1}^{n+1}\right)-A^{-}\left(q_{i+1}^{n+2}-q_{i}^{n+1}\right),
\end{aligned}
$$

with $n$ the relaxation sweep counter and $|A|$ the matrix to be inverted;

$$
|\grave{A}| \equiv A^{+}-A^{-}=\left(\begin{array}{ccc}
c & u & 0 \\
u & c & 0 \\
0 & 0 & u
\end{array}\right) \text {. }
$$

Since $|A|$ is symmetric, the condition number of $|A|$ is determined by the ratio of its largest and smallest eigenvalues. For $0<u<c$, it follows

$$
\Lambda_{|A|}=\operatorname{diag}(c-u, u, u+c) \text {, }
$$

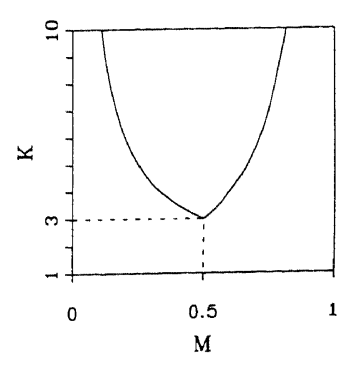

Fig. 1. Condition of the exact, 1-D absolute-eigenvalue matrix as a function of the Mach number. 
and $0, A$ has as condition over the entire subsonic flow regime

$$
K_{n}(M)=\max \left(\frac{1+M}{M}, \frac{1+M}{1-M}\right), \quad M \equiv \frac{|u|}{c} \in(0,1),
$$

see also Fig. 1. The best condition occurs at $M=\frac{1}{2} ; K\left(M=\frac{1}{2}\right)=3$; singularities occur at $M=0$ and $M=1$. Hence, in $1-\mathrm{D}$, in the neighborhood of the static flow condition $(M=0)$, as well as in the neighborhood of the sonic flow condition $(M=1)$, one may expect very large (too large) solution changes in the case of very small right-hand sides only.

In 2-D numerical practice, ill-conditioning of derivative matrices to be inverted is not experienced in the neighborhood of $M=1$, but only near $M=0$. To get some evidence of this we also analyze the 2-D case. With $0<u<c, 0<v<c$, a square finite volume, and $j$ as additional running index in the positive $y$-direction, the following iteration formula is derived for successive downstream and upstream relaxation sweeps:

$$
\begin{aligned}
& (|A|+|B|)\left(q_{i, j}^{n+1}-q_{i, j}^{n}\right) \\
& =-A^{+}\left(q_{i, j}^{n}-q_{i-1, j}^{n+1}\right)-A^{-}\left(q_{i+1, j}^{n}-q_{i, j}^{n}\right)-B^{+}\left(q_{i, j}^{n}-q_{i, j-1}^{n+1}\right)-B^{-}\left(q_{i, j+1}^{n}-q_{i, j}^{n}\right), \\
& (|A|+|B|)\left(q_{i, j}^{n+2}-q_{i, j}^{n+1}\right) \\
& =-A^{+}\left(q_{i, j}^{n+1}-q_{i-1, j}^{n+1}\right)-A^{-}\left(q_{i+1, j}^{n+2}-q_{i, j}^{n+1}\right)-B^{+}\left(q_{i, j}^{n+1}-q_{i, j-1}^{n+1}\right)-B^{-}\left(q_{i, j+1}^{n+2}-q_{i, j}^{n+1}\right),
\end{aligned}
$$

with

$$
\begin{aligned}
& A^{+}=\frac{1}{2}\left(\begin{array}{cccc}
u+c & u+c & 0 & 0 \\
u+c & u+c & 0 & 0 \\
0 & 0 & 2 u & 0 \\
0 & 0 & 0 & 2 u
\end{array}\right), \quad A^{-}=\frac{1}{2}\left(\begin{array}{cccc}
u-c & c-u & 0 & 0 \\
c-u & u-c & 0 & 0 \\
0 & 0 & 0 & 0 \\
0 & 0 & 0 & 0
\end{array}\right), \\
& B^{+}=\frac{1}{2}\left(\begin{array}{cccc}
v+c & 0 & l+c & 0 \\
0 & 2 v & 0 & 0 \\
v+c & 0 & v+c & 0 \\
0 & 0 & 0 & 2 v
\end{array}\right), \quad B^{-}=\frac{1}{2}\left(\begin{array}{cccc}
v-c & 0 & c-v & 0 \\
0 & 0 & 0 & 0 \\
c-v & 0 & v-c & 0 \\
0 & 0 & 0 & 0
\end{array}\right) .
\end{aligned}
$$

Thus, in 2-D the matrix to be inverted is

$$
|A|+|B|=A^{+}-A^{-}+B^{+}-B^{-}=\left(\begin{array}{cccc}
2 c & u & v & 0 \\
u & v+c & 0 & 0 \\
v & 0 & u+c & 0 \\
0 & 0 & 0 & u+v
\end{array}\right) .
$$

When rotating in the flow direction, it follows:

$$
\begin{aligned}
& 1_{A+B}=\operatorname{diag}\left(\bar{u}, \frac{3}{2} c-\frac{1}{2} \sqrt{c^{2}+4 \bar{u}^{2}}, \bar{u}+c, \frac{3}{2} c+\frac{1}{2} \sqrt{c^{2}+4 \bar{u}^{2}}\right), \\
& \bar{u} \equiv u \cos \phi+v \sin \phi, \quad \phi \equiv \arctan \frac{v}{u},
\end{aligned}
$$




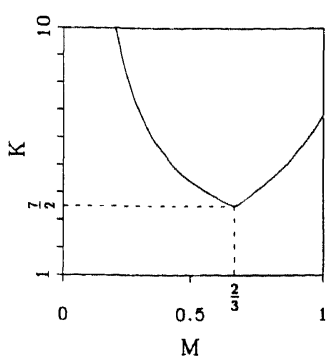

Fig. 2. Condition of the exact, 2-D absolute-eigenvalue matrix as a function of the Mach number.

and thus

$$
K_{|A|+|B|}(M)=\max \left(\frac{3+\sqrt{1+4 M^{2}}}{2 M}, \frac{3+\sqrt{1+4 M^{2}}}{3-\sqrt{1+4 M^{2}}}\right), M \equiv \frac{|\bar{u}|}{c} \in(0,1),
$$

see also Fig. 2. We see that in 2-D the singularity at $M=1$ no longer exists, which explains why the solution method presented in [1] does not fail for transonic flow computations. The best condition occurs at $M=\frac{2}{3}, K\left(\frac{2}{3}\right)=\frac{7}{2}$.

In the remainder of this paper, we discuss possible fixes to the 1-D and 2-D ill-conditionings of the absolute-eigenvalue matrices (Section 2), analyze the multigrid smoothing properties of a favored fix (Section 3) and present some numerical results (Section 4). Our work differs from the conditioning work as reviewed in [6], in that in [6] the conditions of the Jacobians are improved, whereas here the conditions of the absolute-eigenvalue matrices are improved.

\section{Fixes to ill-conditioning of subsonic, absolute-eigenvalue matrices}

\subsection{Trimming 2-D singular matrix}

For 2-D low-Mach-number flows, Eqs. (12a)-(12b) can simply be regularized by (locally) dropping the entropy-equation part, and by replacing it, in the case of e.g. (12a), by either the homentropic iteration formula

$$
s_{i, j}^{n+1}-s_{i, j}^{n} \equiv\left(p_{i, j}^{n+1}-p_{i, j}^{n}\right)-\left(c_{i, j}^{n}\right)^{2}\left(\rho_{i, j}^{n+1}-\rho_{i, j}^{n}\right)=0,
$$

or-alternatively - the incompressible formula

$$
s_{i, j}^{n+1}-s_{i, j}^{n}=p_{i, j}^{n+1}-p_{i, j}^{n} .
$$

If the entropy equation is dropped from system (12a)-(12b), the corresponding derivative matrix to be inverted is reduced to

$$
|A|+|B|=\left(\begin{array}{ccc}
2 c & u & v \\
u & v+c & 0 \\
v & 0 & u+c
\end{array}\right)
$$




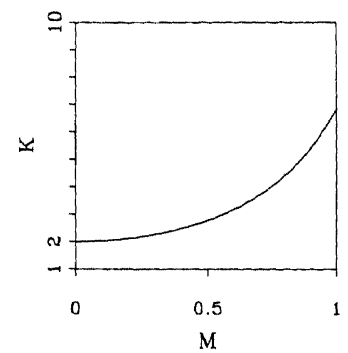

Fig. 3. Condition of the trimmed, 2-D, absolute-eigenvalue matrix as a function of the Mach number.

with, again rotating in the flow direction,

$$
\Lambda_{|A|+|B|}=\operatorname{diag}\left(\frac{3}{2} c-\frac{1}{2} \sqrt{c^{2}+4 \bar{u}^{2}}, \vec{u}+c, \frac{3}{2} c+\frac{1}{2} \sqrt{c^{2}+4 \bar{u}^{2}}\right),
$$

and thus

$$
K_{|A|+|B|}(M)=\frac{3+\sqrt{1+4 M^{2}}}{3-\sqrt{1+4 M^{2}}}, \quad M \in(0,1),
$$

see also Fig. 3. A difficulty in splitting off the singular part from the iteration formulae in the case of general subsonic flows is that it requires the introduction of a monitor for switching on and off homentropy or incompressibility, i.e. (17a) or (17b). Since rigorous formulae for setting thresholds for the monitors are hard to derive, we refrain from applying these reduced derivative matrices.

\subsection{Adding $1-D$ and 2-D regularizing matrices}

The 1-D absolute-eigenvalue matrix (9) can be regularized by adding a matrix $R$ to it, leading to the approximate derivative matrix:

$$
|A|_{R} \equiv|A|+R \text {. }
$$

If we take

$$
R=\alpha\left(\begin{array}{ccc}
0 & -u & 0 \\
0 & 0 & 0 \\
0 & 0 & c-u
\end{array}\right) \quad \text { or } \quad R=\alpha\left(\begin{array}{ccc}
0 & 0 & 0 \\
-u & 0 & 0 \\
0 & 0 & c-u
\end{array}\right)
$$

for any constant $\alpha \in(0,1]$ the singularities at $M=0$ and $M=1$ are removed. Since $|A|_{R}$ is not symmetric, its condition number does not equal the ratio of its largest and smallest eigenvalues. Here we use the general formula $K_{|A|_{R}}=\left\||A|_{R}\right\|\|\||A|_{R}^{-1} \|$. Taking the $L_{\infty}$-norm as the matrix norm, for both $R$ 's in (22) we derive

$$
\begin{aligned}
& K_{|A|_{R}}(M)=\max \left(\frac{1+M}{\alpha+(1-\alpha) M}, \frac{(1+M)^{2}}{1-(1-\alpha) M^{2}}\right), \\
& M \in(0,1), \quad \alpha \in(0,1],
\end{aligned}
$$




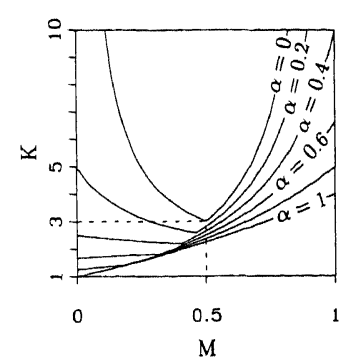

Fig. 4. Condition of the through addition regularized, 1-D absolute-eigenvalue matrix, as a function of the Mach number.

see also Fig. 4. For $\alpha=1,|A|_{R}$ is best conditioned over the entire subsonic Mach-number range, while the corresponding approximate derivative matrix $|A|_{R}$ will generally be rather close to the exact derivative matrix $|A|$. A convergence requirement to be satisfied is that the eigenvalues of $|A|_{R}$ are positive. This requirement is met by both $R$ 's from (22), for any $\alpha \in(0,1]$.

In 2-D, where no sonic singularity exists, we may take the symmetric matrix

$$
R=\alpha\left(\begin{array}{cccc}
0 & 0 & 0 & 0 \\
0 & 0 & 0 & 0 \\
0 & 0 & 0 & 0 \\
0 & 0 & 0 & c-u-v
\end{array}\right), \quad \alpha \in(0,1]
$$

to regularize (14). Thus, in 2-D we have the advantage that we remain closer to the exact derivative matrix than we do in 1-D. For the corresponding eigenvalue-matrix $(|A|+|B|)_{R} \equiv$ $|A|+|B|+R$ it follows that

$$
\Lambda_{(|A|+|B|)_{R}}=\operatorname{diag}\left(\alpha c+(1-\alpha) \bar{u}, \frac{3}{2} c-\frac{1}{2} \sqrt{c^{2}+4 \bar{u}^{2}}, \bar{u}+c, \frac{3}{2} c+\frac{1}{2} \sqrt{c^{2}+4 \bar{u}^{2}}\right),
$$

and hence

$$
K_{(|A|+|B|)_{R}}(M)=\max \left(\frac{3+\sqrt{1+4 M^{2}}}{2(\alpha+(1-\alpha) M)}, \frac{3+\sqrt{1+4 M^{2}}}{3-\sqrt{1+4 M^{2}}}\right), \quad M \in(0,1), \quad \alpha \in(0,1],
$$

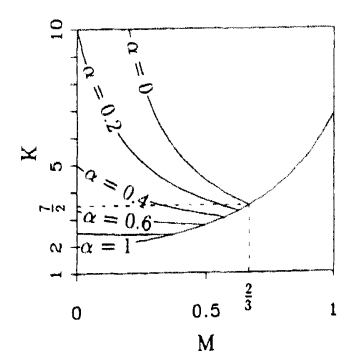

Fig. 5. Condition of the through addition regularized, 2-D absolute-eigenvalue matrix as a function of the Mach number. 
see also Fig. 5. In the next section, for 2-D flows, we will investigate the multi-grid smoothing (high-frequency damping) properties of point Gauss-Seidel relaxation when additive conditioning is applied. For reasons of simplicity, smoothing properties are investigated for the 1-D equation.

\section{Smoothing analysis of additive conditioning}

Consider the downstream iteration formula

$$
(|A|+R)\left(q_{i}^{n+1}-q_{i}^{n}\right)=-A^{+}\left(q_{i}^{n}-q_{i-1}^{n+1}\right)-A^{-}\left(q_{i+1}^{n}-q_{i}^{n}\right),
$$

and the upstream formula

$$
(|A|+R)\left(q_{i}^{n+2}-q_{i}^{n+1}\right)=-A^{+}\left(q_{i}^{n+1}-q_{i-1}^{n+1}\right)-A^{-}\left(q_{i+1}^{n+2}-q_{i}^{n+1}\right),
$$

where $R$ is the 1-D equivalent of the 2-D additive matrix (24):

$$
R=\alpha\left(\begin{array}{ccc}
0 & 0 & 0 \\
0 & 0 & 0 \\
0 & 0 & c-u
\end{array}\right) .
$$

In order to investigate the smoothing properties, the local solution error

$$
\delta_{i}^{n}=q_{i}^{*}-q_{i}^{n}
$$

and its Fourier form

$$
\delta_{i}^{n}=D^{n} \mathrm{e}^{\mathrm{i} \theta}, \quad|\theta| \in\left[\frac{1}{2} \pi, \pi\right]
$$

are introduced, where $q_{i}^{*}$ is the exact local solution, $D^{n}$ the amplitude vector $\left(D_{1}^{n}, D_{2}^{n}, D_{3}^{n}\right)$ and $\mathrm{e}^{\mathrm{i} \theta}$ the (scalar) mode. If the coefficient matrices in (27a) and (27b) are kept frozen, it follows from (27a) and (27b) for the corresponding amplification matrices $\mathscr{M}_{\text {downstream }}$ and $\mathscr{M}_{\text {upstream: }}$ :

$$
\begin{aligned}
& \mathscr{M}_{\text {downstream }}=\left(\left(1-\mathrm{e}^{-\mathrm{i} \theta}\right) A^{+}-A^{-}+R\right)^{-1}\left(-\mathrm{e}^{\mathrm{i} \theta} A^{-}+R\right), \\
& \mathscr{M}_{\text {upstream }}=\left(A^{+}-\left(1-\mathrm{e}^{\mathrm{i} \theta}\right) A^{-}+R\right)^{-1}\left(\mathrm{e}^{-\mathrm{i} \theta} A^{+}+R\right) .
\end{aligned}
$$

In both $\mathscr{M}_{\text {downstream }}$ and $\mathscr{M}_{\text {upstream }}$ the influence of $\alpha$ is confined to a single eigenvalue per matrix only:

$$
\begin{aligned}
& \lambda_{\mathscr{M}_{\mathrm{downstream}}}(\alpha)=\frac{\alpha(1-M)}{\alpha(1-M)+\left(1-\mathrm{e}^{-\mathrm{i} \theta}\right) M}, \\
& \lambda_{. / \text {upstream }}(\alpha)=\frac{\alpha(1-M)+\mathrm{e}^{-\mathrm{i} \theta} M}{\alpha(1-M)+M} .
\end{aligned}
$$

It can be seen that for $\alpha=1$, it still holds that

$$
\left|\lambda_{\mathscr{M}_{\text {downstream }}}(1)\right| \leqslant 1 \quad \text { and }\left|\lambda_{\mathscr{M}_{\text {upstream }}}(1)\right| \leqslant 1, \quad \forall|\theta| \in\left[\frac{1}{2} \pi, \pi\right], \quad \forall M \in(0,1) \text {. }
$$


We assume that $\alpha=1$ is an acceptable choice in 2-D as well. In the next section the conservative implementation of the 2-D additive conditioning is discussed and some numerical results are given for a 2-D stagnation flow.

\section{Application of additive conditioning}

\subsection{2-D conservative implementation}

If we discretize the steady, 2-D, conservative Euler equations by a first-order upwind, cell-centered finite-volume method, and denote the numerical flux functions which approximate the cell-face fluxes in $x$ - and $y$-direction $\left(f\left(q_{i+1 / 2, j}\right)\right.$ and $\left.g\left(q_{i, j+1 / 2}\right)\right)$ by $F\left(q_{i, j}, q_{i+1, j}\right)$ and $G\left(q_{i, j}, q_{i, j+1}\right)$, respectively, the conservative upstream and downstream relaxation sweeps read:

$$
\begin{aligned}
& {\left[\frac{\partial F\left(q_{i, j}^{n}, q_{i+1, j}^{n}\right)}{\partial q_{i, j}^{n}} h_{i+1 / 2, j}-\frac{\partial F\left(q_{i-1, j}^{n+1}, q_{i, j}^{n}\right)}{\partial q_{i, j}^{n}} h_{i-1 / 2, j}+\frac{\partial G\left(q_{i, j}^{n}, q_{i, j+1}^{n}\right)}{\partial q_{i, j}^{n}} h_{i, j+1 / 2}\right.} \\
& \left.-\frac{\partial G\left(q_{i, j-1}^{n+1}, q_{i, j}^{n}\right)}{\partial q_{i, j}^{n}} h_{i, j-1 / 2}+\frac{\mathrm{d} Q}{\mathrm{~d} q}\left(q_{i, j}^{n}\right) R\left(q_{i, j}^{n}\right) \bar{h}_{i, j}\right]\left(q_{i, j}^{n+1}-q_{i, j}^{n}\right) \\
& =F\left(q_{i-1, j}^{n+1}, q_{i, j}^{n}\right) h_{i-1 / 2, j}-F\left(q_{i, j}^{n}, q_{i+1, j}^{n}\right) h_{i+1 / 2, j}+G\left(q_{i, j-1}^{n+1}, q_{i, j}^{n}\right) h_{i, j-1 / 2} \\
& -G\left(q_{i, j}^{n}, q_{i, j+1}^{n}\right) h_{i, j+1 / 2} \text {, } \\
& {\left[\frac{\partial F\left(q_{i, j}^{n+1}, q_{i+1, j}^{n+2}\right)}{\partial q_{i, j}^{n+1}} h_{i+1 / 2, j}-\frac{\partial F\left(q_{i-1, j}^{n+1}, q_{i, j}^{n+1}\right)}{\partial q_{i, j}^{n+1}} h_{i-1 / 2, j}+\frac{\partial G\left(q_{i, j}^{n+1}, q_{i, j+1}^{n+2}\right)}{\partial q_{i, j}^{n+1}} h_{i, j+1 / 2}\right.} \\
& \left.-\frac{\partial G\left(q_{i, j-1}^{n+1}, q_{i, j}^{n+1}\right)}{\partial q_{i, j}^{n+1}} h_{i, j-1 / 2}+\frac{\mathrm{d} Q}{\mathrm{~d} q}\left(q_{i, j}^{n+1}\right) R\left(q_{i, j}^{n+1}\right) \bar{h}_{i, j}\right]\left(q_{i, j}^{n+2}-q_{i, j}^{n+1}\right) \\
& =F\left(q_{i-1, j}^{n+1}, q_{i, j}^{n+1}\right) h_{i-1 / 2, j}-F\left(q_{i, j}^{n+1}, q_{i+1, j}^{n+2}\right) h_{i+1 / 2, j}+G\left(q_{i, j-1}^{n+1}, q_{i, j}^{n+1}\right) h_{i, j-1 / 2} \\
& -G\left(q_{i, j}^{n+1}, q_{i, j+1}^{n+2}\right) h_{i, j+1 / 2},
\end{aligned}
$$

where $\bar{h}_{i, j}$ is a cell-averaged mesh width, e.g. $\bar{h}_{i, j}=\frac{1}{4}\left(h_{i-1 / 2, j}+h_{i+1 / 2, j}+h_{i, j-1 / 2}+h_{i, j+1 / 2}\right)$, and where

$$
\frac{\mathrm{d} Q}{\mathrm{~d} q}=\left(\begin{array}{cccc}
\frac{\rho}{c} & 0 & 0 & -\frac{1}{c^{2}} \\
\frac{\rho u}{c} & \rho & 0 & -\frac{u}{c^{2}} \\
\frac{\rho v}{c} & 0 & \rho & -\frac{v}{c^{2}} \\
\frac{1}{2} \frac{\rho\left(u^{2}+v^{2}\right)}{c}+\frac{1}{\gamma-1} \rho c & \rho u & \rho v & -\frac{1}{2} \frac{u^{2}+v^{2}}{c^{2}}
\end{array}\right) .
$$




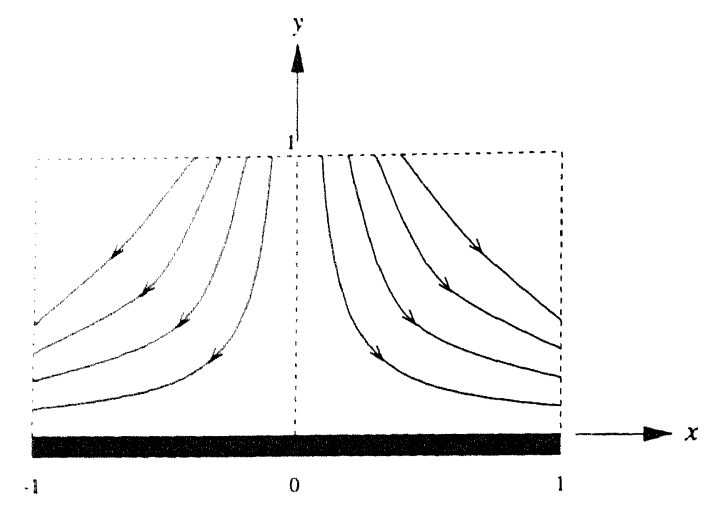

Fig. 6. Stagnation flow against a flat plate.

With (24) and $\alpha=1$, we get

$$
\frac{\mathrm{d} Q}{\mathrm{~d} q} R=\left(\begin{array}{cccc}
0 & 0 & 0 & -\frac{c-u-v}{c^{2}} \\
0 & 0 & 0 & -\frac{u(c-u-v)}{c^{2}} \\
0 & 0 & 0 & -\frac{v^{2}(c-u-v)}{c^{2}} \\
0 & 0 & 0 & -\frac{1}{2} \frac{\left(u^{2}+c^{2}\right)(c-u-v)}{c^{2}}
\end{array}\right)
$$

\subsection{Numerical results}

A suitable test case is steady, 2-D stagnation flow normal to a flat plate (Fig. 6). A favorable property of this test case is the direct availability of good approximate boundary conditions (because of the availability of an exact, incompressible potential flow solution, see e.g. [4, Chapter X]). For computational efficiency, we only compute the half problem $(x \geqslant 0)$. Note that exact solutions of subsonic flows tangential to a kinked wall have a singularity at the kink for all kink angles $\delta$ except $\delta=\frac{1}{2} \pi$ (see e.g. [7, Section 4.1]), which case is identical to the present normal stagnation flow. We introduce a reference speed $w_{\text {ref }}$, a reference density $\rho_{\text {ref }}$ and a reference Mach number $M_{\text {ref }}$ as the known quantities in the point $(x, y)=(1,1)$. Then the boundary conditions imposed are:

- at the inflow boundary, assuming homenthalpy:

$$
\begin{aligned}
& u(x, 1)=w_{\mathrm{ref}} x, \\
& l(x, 1)=-w_{\mathrm{ref}}, \\
& c(x, 1)=\sqrt{\frac{w_{\mathrm{ref}}^{2}}{M_{\mathrm{ref}}^{2}}+\frac{\gamma-1}{2}\left(w_{\mathrm{ref}}^{2}-u^{2}(x, 1)-v^{2}(x, 1)\right)}
\end{aligned}
$$



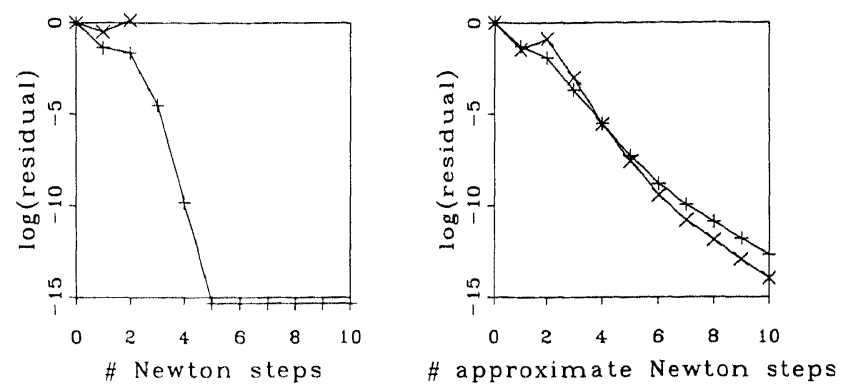

Fig. 7. Convergence behaviors of point relaxation, $+: M_{\text {ref }}=0.1, \times: M_{\text {ref }}=0.075$. (a) Without conditioning. (b) With conditioning.

- at the outflow boundary, assuming homentropy and homenthalpy:

$$
p(1, y)=\left(1+\frac{\gamma-1}{2} \frac{w^{2}(1, y)}{c^{2}(1, y)}\right)^{-\gamma /(\gamma-1)} p_{t},
$$

where

$$
\begin{aligned}
& w^{2}(1, y)=w_{\mathrm{ref}}^{2}\left(1+y^{2}\right), \\
& c^{2}(1, y)=\frac{w_{\mathrm{ref}}^{2}}{M_{\mathrm{ref}}^{2}}+\frac{\gamma-1}{2}\left(w_{\mathrm{ref}}^{2}-w^{2}(1, y)\right), \\
& p_{t}=\left(1+\frac{\gamma-1}{2} M_{\mathrm{ref}}^{2}\right)^{\gamma /(\gamma-1)} p_{\mathrm{ref}}, \quad p_{\mathrm{ref}}=\frac{1}{\gamma} \frac{w_{\mathrm{ref}}^{2}}{M_{\mathrm{ref}}^{2}} \rho_{\mathrm{ref}} ;
\end{aligned}
$$

- at the vertical-wall boundary:

$$
u(0, y)=0
$$

- at the lower-wall boundary:

$$
v(x, 0)=0 \text {. }
$$

In Fig. 7 for two low-subsonic (though not yet very low-subsonic) values of $M_{\text {ref }}$, we give the convergence behavior of the point relaxation in some arbitrary cell, at some arbitrary instant in the iteration process. (The residual considered is that of the energy equation.) From the results it appears that the additive conditioning does a good job. Though quadratic convergence is lost, the divergence which occurs at $M_{\text {ref }}=0.075$ (Fig. 7(a)) has disappeared by application of the conditioning (Fig. 7(b)). In Fig. 8, convergence results are presented, as obtained by means of the conditioned relaxation method accelerated by nonlinear multigrid. (The residual considered is the $L_{\infty}$-norm of the energy equation's residual field.) The Mach-number sequence considered is: $M_{\text {ref }}=0.5,0.05,0.005$. Note that the method does not break down, but still converges in the very low-subsonic case $M_{\text {ref }}=0.005$. The decrease of convergence rates at decreasing Mach numbers that can be observed, is related to the decreasing entropy-error convection across domain boundaries. Fixing this would require the condition improvement of the Jacobians. As 


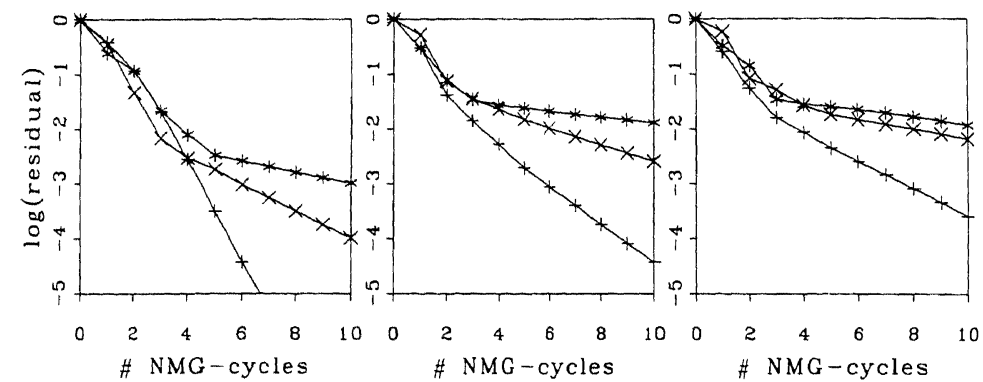

Fig. 8. Convergence behaviors of nonlinear multigrid iteration, $+: h=1 / 8, \quad \times: h=1 / 16, *: h=1 / 32$. (a) $M_{\text {ref }}=0.5$. (b) $M_{\text {ref }}=0.05$. (c) $M_{\text {ref }}=0.005$.

mentioned, a review of such techniques is given in [6]. (An early research paper in the context of single-grid, explicit time-stepping schemes is [8].)

\section{Conclusions}

Two methods have been proposed for removing singularities in local, absolute-eigenvalue matrices of upwind-discretized Euler equations:

- elimination of the entropy-equation part from the exact, 2-D derivative matrix,

- addition of a singular matrix (which is very close to the zero matrix) to the singular, exact derivative matrix.

The first fix is insufficient in 1-D, and also has the drawback that its successful application requires tuning. The second fix works in all three dimensions, is free of tuning parameters and may remove the ill-conditioning without deteriorating too much the quadratic convergence rate of exact Newton iteration. The latter fix has been successfully applied to a steady, 2-D, low-subsonic stagnation flow.

\section{Acknowledgment}

Mrs. Joke Sterringa and the referees are acknowledged for their suggestions in improving this paper.

\section{References}

[1] P.W. Hemker and S.P. Spekreijse, Multiple grid and Osher's scheme for the efficient solution of the steady Euler equations, Appl. Numer. Math. 2 (1986) 475-493.

[2] B. Koren, Defect correction and multigrid for an efficient and accurate computation of airfoil flows, J. Comput. Phys. 77 (1988) 183-206. 
B. Koren and P.W. Hemker, Damped, direction-dependent multigrid for hypersonic flow computations, Appl. Numer. Math. 7 (1991) 309-328.

L. Prandtl and O.G. Tietjens, Fundamentals of Hydro- and Aeromechanics (Dover, New York, 1957).

E. Turkel, Preconditioned methods for solving the incompressible and low speed compressible equations, $J$. Comput. Phys. 72 (1987) 277-298.

E. Turkel, Review of preconditioning methods for fluid dynamics, Appl. Numer. Math. 12 (1993) 257-284.

$\mathrm{E}$. van der Maarel and B. Koren, Spurious, zeroth-order entropy generation along a kinked wall, Int. J. Numer. Meth. Fluids 13 (1991) 1113-1129.

B. van Leer, W.-T. Lee and P.L. Roe, Characteristic time-stepping or local preconditioning of the Euler equations, AIAA-91-1552 (1991). 